\title{
Capacidades profesionales para el mañana de la comunicación estratégica: contribuciones desde España y Argentina
}

\author{
Professional capabilities for the future of strategic \\ communication: Contributions from Spain and Argentina
}

\author{
Gutiérrez-García, E. y Sadi, G. 1 \\ Recibido: 30-09-2019 - Aceptado: 24-01-2020 \\ DOI: https://doi.org/10.26441/RC19.1-2020-A8
}

\begin{abstract}
RESUMEN: El Global Capability Framework for Public Relations and Communication Management (Fawkes et at., 2018) es la primera propuesta internacional que adopta el concepto de 'capacidad' en la disciplina para describir el alcance y potencial futuro de una profesión cambiante y global. El resultado, un marco global de 11 capacidades, producto de una investigación académica bienal (Delphi, encuestas, grupos focales y entrevistas) en la que participaron más de 1.400 expertos académicos y profesionales en nueve países de cinco continentes (Argentina, Australia, Canadá, España, Estados Unidos, Reino Unido, Singapur, Suecia y Sudáfrica).

Dado que cada país cuenta con su propio marco de capacidades, en este artículo se presentan de forma inédita los marcos específicos de Argentina y España -únicos países de habla hispana en el proyecto- con sus listas de capacidades y análisis comparado. Como conclusión general, se revela una marcada orientación estratégica y social de la profesión, a la vez que se evidencian algunas diferencias significativas.
\end{abstract}

Palabras clave: capacidades profesionales; competencias; relaciones públicas; comunicación estratégica; Argentina; España.

ABSTRACT: The Global Capability Framework for Public Relations and Communication Management (Fawkes et at., 2018) is the first international proposal that applies the capability concept to the discipline in order to describe the scope and future potential of a global profession. A global framework with 11 capabilities is the result of an empirical research (Delphi, survey, interviews and focus groups) with more than 1.400 experts, scholars and practitioners, in nine countries of five continents (Argentina, Australia, Canada, Singapore, South Africa, Spain, Sweden, UK and USA).

Also, each country has its own capability framework. In this paper it is presented for the first time the specific frameworks, and its comparison, from Argentina and Spain, the only participants' hispanic countries. As a general conclusion, the findings reveal a strong strategic and social orientation of the profession, but there are significant differences that may be explained by different regional contexts.

Keywords: professional capabilities; public relations; strategic communication; competencies; Argentina; Spain.

\footnotetext{
${ }^{1}$ Elena Gutiérrez-García es Doctora en Comunicación por la Universidad de Navarra y Profesora de Gestión de Proyectos, Dirección de Comunicación Empresarial y Stakeholders Management en la Facultad de Comunicación de la misma Universidad. Es Directora del Grupo de Investigación interdisciplinar INCOMIN y Miembro de la Junta Directiva de la Asociación Española de Directivos de Comunicación. egutierrez@unav.es, https://orcid.org/0000-0001-6740-7014

Gabriel Sadi es Doctor en Comunicación Social por la Universidad Austral y magister en Comunicación e Imagen Institucional por la UCAECE. Lecturer, Department of Management, Huddersfield Business School, University of Huddersfield (Reino Unido). International Liaison Representative 2020-2022, PR Division, International Communication Association. Contacto nacional Argentina y Uruguay, Latin American Communication Monitor. g.sadi@hud. ac.uk, https://orcid.org/0000-0001-8675-5735
} 


\section{Introducción ${ }^{2}$}

La disciplina de la comunicación estratégica/relaciones públicas y su práctica profesional han heredado una ambivalencia conceptual desde el siglo XX, causada por la diversidad léxica, su riqueza práctica, y acentuada por la falta de consenso sobre su ámbito y objeto de estudio formal (Fawkes, Gregory y Montoya-Martínez, 2017a; Nothhaft, 2016; Sadi, 2014; Sandhu, 2017). Esta situación, sin duda, tiene consecuencias en cómo se definen los roles y competencias profesionales, $\mathrm{y}$ ha sido uno de los temas constantes de investigación académica, con estudios pioneros como los de Broom y Dozier (1986) o Broom y Smith (1979), al igual que el debate sobre la adaptación de la oferta universitaria (ver los informes publicados por The Commission on PR Education, 2017). Al mismo tiempo, este debate ha estado acompañado de un interés por explicar cuál es la identidad profesional y cómo legitimarla socialmente (Edwards, 2018: 146-157); hecho muy relacionado a cómo deberían sus profesionales ejercer de modo competente la profesión.

Por otro lado, la evolución práctica y el desarrollo teórico evidencian que nos encontramos ante una disciplina y profesión con una gran expansión en diferentes regiones geográficas. Esta realidad induce a cuestionarnos cuáles son las características profesionales compartidas de una profesión ya global, aun respetando las diferencias culturales y regionales. Por otro lado, la disciplina sigue teniendo una marcada influencia occidental y anglosajona (Gregory y Fawkes, 2019; Manley y Valin, 2017; Tench et al., 2013; Sriramesh y Verçiç, 2009).

La pregunta fundamental, por tanto, continúa siendo qué caracteriza a una profesión global como las relaciones públicas y la comunicación estratégica; y más concretamente, qué capacita a sus profesionales para afrontar los desafíos futuros de una profesión cambiante. Las investigaciones hasta la fecha se han centrado en las competencias del presente, y no tanto en el enfoque sobre el potencial futuro. Al respecto, uno de los estudios recientes es el GBOK (Global Body of Knowledge) (Manley y Valin, 2017), impulsado por la Global Alliance for Public Relations and Communication Management -en adelante Global Alliance-. El resultado, un listado con cientos de descripciones sobre el denominado KSAB (Knowledge, Skills, Attributes, Behaviors) -conocimientos, habilidades, atributos y comportamientos-, según niveles de experiencia profesional, que van desde el nivel de entrada hasta el de mayor seniority. Al GBOK se llegó tras el análisis de 31 fuentes (marcos competenciales en varios países e investigaciones académicas).

Se da por hecho que los profesionales serán competentes en las áreas que abarcan las KSAB (y según el nivel de experiencia). Sin embargo, la realidad parece demostrar que los profesionales dicen haber utilizado diferentes conocimientos, habilidades, atributos y comportamientos en cada etapa de su carrera y dependiendo de las necesidades de su contexto. En definitiva, las KSAB no están orientadas al futuro, sino a lo que se necesita para ser competente en el presente (Gregory, 2008; Moreno, Tench y Okay, 2017; Tench et al., 2013).

La realidad referida en esta introducción induce a preguntarse cómo pueden los profesionales capacitarse para afrontar los desafíos futuros. Es decir, se propone ampliar el foco, para aportar una reflexión sobre cuál es la prospectiva o el potencial al que están llamados sus profesionales; en de-

\footnotetext{
${ }^{2}$ Los autores expresan su agradecimiento al equipo de investigadores del proyecto global, en especial a las directoras del proyecto Dra. Anne Gregory y Dra. Johanna Fawkes. Al Consejo Profesional de Relaciones Públicas de la República Argentina y la Asociación de Directivos de Comunicación de España. En España, a los profesores Mónica Recalde, Ángel Losada y Enrique Sueiro; junto a las ayudantes de investigación Lucía Gutiérrez y Natalia Celis. En Argentina, a los investigadores asistentes Verónica Méndez, Evelyn Moyano, Luciana Accorsi, Leonardo Arana y Leonardo Mancusi. Agradecimiento especial a la colaboración de más de 100 académicos, empleadores y profesionales de ambos países.
} 
finitiva, lograrlo mediante la adopción del concepto de capacidad y su concreción en la formulación de una lista específica de capacidades.

Es ésta justamente la perspectiva conceptual de este artículo: 'capacidad', traducción del término inglés capability, que, sin embargo, no ha sido adoptado hasta ahora en nuestra disciplina, pero que sí cuenta con aplicación más extendida en otras profesiones, como recientemente han mencionado investigadores de la comunicación (Macnamara, 2018; Macnamara et al., 2018; Thurlow et al., 2018). En virtud de ello, la investigación internacional en la que se enmarca este artículo, el proyecto Global Capability Framework (GCF), ha dado como resultado por primera vez un marco global de capacidades, el Global Capability Framework for Public Relations and Communication Management (Fawkes et al., 2018). Este marco resultante describe la profesión globalmente con 11 capacidades, gracias a una investigación académica (Delphi, encuestas, grupos focales y entrevistas) en la que participaron más de 1.400 expertos académicos y profesionales en nueve países de cinco continentes (Argentina, Australia, Canadá, España, Estados Unidos, Reino Unido, Singapur, Suecia y Sudáfrica) (véase el detalle sobre cómo se desarrolló la investigación y el marco global resultante en el artículo en Public Relations Review de Gregory y Fawkes, 2019).

Como punto de referencia de este artículo, el proyecto internacional GCF ha dado como resultado, también, nueve marcos de capacidades en cada uno de los países participantes. En el contexto de esta investigación global, el presente artículo presenta de forma inédita, agrupados y comparados por primera vez, los principales hallazgos de los marcos específicos de Argentina y España, únicos países del proyecto, además, de habla hispana. Para contextualizar cómo se llegó al marco de capacidades específico de cada país, el objetivo es centrarnos en la descripción detallada sobre la fase de investigación cualitativa (paneles Delphi y grupos de discusión), a fin de ayudar a comprender cómo se construyeron sendos marcos mediante un diálogo abierto y reflexivo con un total de 107 expertos profesionales y académicos seleccionados en la muestra del estudio en ambos países.

Por ello, en los siguientes apartados se ofrece, primero, un marco teórico que contextualiza los antecedentes sobre el concepto de competencias, para seguidamente realizar una síntesis acerca de la noción de capacidad. El concepto se ha adoptado con perspectiva interdisciplinar, procedente de la teoría del desarrollo humano. Seguidamente, se apunta cómo se ha ido extendiendo también desde finales del siglo XX en los estudios profesionales. Tras una breve incursión en sendas disciplinas, se presenta una síntesis de los antecedentes sobre el estudio de las competencias en las relaciones públicas y la comunicación estratégica; antecedente conceptual más inmediato del concepto de capacidad, a fin de comprender por qué su propuesta en nuestra disciplina contribuye a dar un paso más en la prospectiva profesional. Es decir, en lugar de seguir construyendo un marco de trabajo en el que se ofrecen descripciones exhaustivas de las competencias y sus elementos KSAB (conocimientos, habilidades, atributos y comportamientos), se establecen marcos de capacidades con un número de enunciados menor, holístico y flexible, pero que describen el alcance de la profesión, a fin de aspirar a lograr un estándar profesional.

En segundo lugar, el apartado metodológico específico en Argentina y España presenta consideraciones sobre sus respectivos contextos profesionales, para ayudar a comprender el porqué de los marcos resultantes. En el cuarto se ofrecen los principales hallazgos por separado de ambos países para, por último, en el quinto apartado realizar un análisis comparado que apunte y advierta las similitudes y diferencias entre los marcos resultantes de Argentina y España.

\section{Marco teórico: antecedentes del concepto y aplicación a la disciplina}

La noción de capacidad ha ganado protagonismo en diversas disciplinas, y ofrece una perspectiva centrada en el potencial futuro de los profesionales, agrandando así la perspectiva competencial. 
En este apartado se ofrece una síntesis que, por limitaciones de espacio, es breve y no exhaustiva, a fin de priorizar la explicación de cómo se adoptó el concepto en nuestra disciplina y cómo se aplicó una metodología empírica para implicar a los principales actores -profesionales y académicos- y posibilitar la concreción del marco de capacidades.

El propósito de esta revisión del marco teórico no es otro que explicar qué línea de pensamiento rodea a la noción de capacidad y cuáles son sus fundamentos. El concepto ha ganado auge desde los años 80 en los estudios profesionales de diversas disciplinas, como un modo de diferenciarse respecto al de competencia. Éste tiene que ver con lo que una persona debe ser capaz de hacer en el momento presente para desarrollar su ejercicio profesional excelente (Lester, 2014). En 1980, la Royal Society of Arts publicó en Londres un manifiesto que defendía reflexionar sobre las capacidades, porque parecía más adecuado para enfrentarse a los rápidos cambios. Sólo así, se argüía, se lograrían integrar adecuadamente y en perspectiva los conocimientos, habilidades y cualidades personales, base sobre la que se erige el concepto de competencia. En este sentido, Stephenson señala que el concepto de "capacidad implica competencia, pero mira hacia el futuro, al potencial. Por tanto, este foco supone analizar la capacidad de las personas para participar en la formulación de sus propias necesidades de desarrollo, y siempre en el contexto en que trabajan y viven" (1998: 3).

Al igual que a finales del siglo XX se inició el debate en los estudios profesionales y de educación superior, igualmente se produjo en la teoría de desarrollo humano. Robeyns (2005) ofrece un análisis sobre los antecedentes, basado en las propuestas del economista premio Nobel Amartya Sen y la filósofa Martha Nussbaum. Ambos han propugnado que las políticas públicas deberían virar hacia otro paradigma de indicadores de desarrollo humano, con perspectiva humanista, poniendo en el centro a la persona y su potencial, es decir, sus capacidades, lo que implica reconocer también cómo les afectarán a las personas las limitaciones y las potencialidades del contexto en el que viven (Robeyns, 2005; Sen, 1993). A partir de estas ideas, han aflorado estudios sobre el papel de la formación superior y la gestión del talento en las organizaciones.

Desde el concepto de capacidad se trata de definir las profesiones y su potencial, su esencia, más allá de las normativas y descriptivas listas de niveles competenciales en el presente. En este sentido, existen observaciones de varios autores desde la perspectiva del profesionalismo (véase Stephenson, 1998), como el destacado autor Stan Lester, quien viene cuestionando un exclusivo enfoque competencial. Poniendo en el centro a la persona, Lester (2014) destaca que para hallar estándares en una profesión se necesita avanzar hacia la noción de capacidad. A su juicio, esta noción extiende la de competencia y la contextualiza, pone el énfasis en las oportunidades del profesional para alcanzar sus competencias, y remite a un contexto, pues incluye considerar las limitaciones para lograrlas (por ejemplo, acceso a recursos, formación o tecnología), no siempre contempladas en un análisis competencial.

No obstante, aunque la noción de capacidad también analiza el qué se debe poder hacer, lo hace con otros matices, como es "tener el potencial de ser competente, pero menos normativo o prescriptivo (...) y yendo más allá de la descripción” (Lester, 2014: 37). En este contexto, surgió un movimiento en los años 90, UK Higher Education for Capability (Lester, 2014). Lester investigó a más de 54 profesiones e identificó sus competencias, distinguiendo entre aquellas que describen los atributos necesarios de las personas que entran a una profesión, junto a las tareas o funcionalidades que deben desempeñar en su ejercicio profesional (Lester, 2009). En su análisis del profesional capaz, O’Reilly, Cunningham y Lester (1999) incluyen la habilidad de ir más allá de lo que se considera competente. Por ello, el concepto capacidad ofrece un "modelo abierto" y más flexible (Lester, 2014: 38), trascendiendo la descripción de funciones y funcionalidades. Fawkes et al. (2017b), al respecto, señalan cuando proponen adoptar este concepto a la comunicación: 
"Los planteamientos y redacción sobre capacidades profesionales tienden a ser más fluidos, dinámicos y con la perspectiva de asuntos más generales de la profesionalización, como por ejemplo la capacidad de reflexionar críticamente o aplicar un juicio independiente en situaciones complejas" (p. 15).

\section{De las competencias a las capacidades en la comunicación}

En este apartado se resumen las principales aportaciones desde el ámbito específico de las relaciones públicas y la comunicación estratégica. El antecedente más significativo lo encontramos en 1979, con la investigación de Broom y Smith sobre la consultoría en Estados Unidos. Los autores se cuestionaron por qué la profesión abarcaba funcionalidades tan diversas, desde la escritura hasta el asesoramiento directivo (1979: 48-53). Siguiendo esta línea de investigación, uno de los estudios posteriores y más influyentes es el de la teoría de la excelencia de las relaciones públicas, presentado por Dozier (1992), quien sintetizó la esencia de los roles profesionales en dos: técnico y gestor. DeSanto y Moss (2005) y Moss, Newman y DeSanto (2005) han ahondado en los enfoques gestores/directivos en los Estados Unidos y el Reino Unido. Por su parte, Gregory (2008) realizó una investigación en los sectores británicos público y privado, a fin de observar similitudes y diferencias entre ambos. La autora se centra en la perspectiva estratégica, y aplica el Universal Competency Framework de Bartram (2005), llegando a 10 competencias clave en cada sector (Gregory, 2008: 217-220).

Como se observa, otro elemento clave en el corpus doctrinal sobre roles profesionales es cuestionarse cuáles son las competencias de quienes los ocupan; aunque con una marcada perspectiva anglosajona y sin que exista aún una propuesta internacional o global consensuada. Al respecto, en la última década se han publicado hallazgos que pretenden ser más globales, destacando el proyecto europeo ECOPSI y el global GBOK.

ECOPSI (European Communication Professional Skills and Innovation Programme) combina datos del estudio longitudinal del European Communication Monitor (ECM) en más de 40 países, con focus groups y 53 entrevistas a profesionales de siete países europeos (Tench et al., 2013). Su conclusión: la propuesta del modelo competencial COMPAS (Counselling, Organising/executing, Managing, Performing/creating, Analyzing/interpreting y Supporting/guiding), que integra una matriz de cuatro roles gestores - dirección, crisis, interna y digital-, con su correspondiente detallado número de competencias. Como señalan Fawkes et al. (2017b), modelos competenciales como el ECOPSI habitualmente "tienden a ser operativos y focalizados en competencias funcionales" (p. 8).

En el caso del GBOK (Global Body of Knowledge), promovido por la federación mundial Global Alliance, las competencias se analizaron según los niveles de experiencia: desde niveles de entrada hasta seniority o de dirección. Tuvo como fin lograr un análisis comparado y similitudes entre los diversos países (aunque prevaleció la orientación anglosajona); y el resultado fue un listado con cientos de conocimientos, habilidades, actitudes y comportamientos profesionales globales: la llamada KSAB list (Knowledge, Skills, Attitudes and Behaviors) (GBOK Project, 2015; Manley y Valin, 2017). Tras la presentación del GBOK, en un congreso de investigación de la Global Alliance -el World PR Forum-, se impulsó ampliar la perspectiva de las competencias, dando lugar al proyecto Global Capability Framework (Global Alliance, 2016).

Las investigaciones precedentes permiten concluir que en las últimas cuatro décadas se ha logrado definir con más precisión las competencias profesionales y sus funcionalidades; si bien, como se ha referido, con escaso enfoque global y marcado sesgo anglosajón (Berkowitz y Hristodoulakis, 1999; Broom y Smith, 1979; Dozier y Broom, 2006). Igualmente, sigue habiendo una laguna de conocimiento sobre cómo definir el potencial y, por tanto, capacidad del profesional del futuro, más allá de las funcionalidades y roles específicos, por otro lado cambiantes. 


\section{Metodología}

Dado que la meta de la investigación era alcanzar un marco global consensuado de capacidades profesionales, los investigadores diseñaron una metodología empírica con criterios comunes, aplicable en los nueve países, a fin de lograr el posterior análisis comparado que permitió emerger al marco global propuesto (véanse los detalles en el artículo citado de Gregory y Fawkes, 2019).

Este apartado se centra en describir la aplicación de la fase cualitativa en Argentina y España, con sendos estudios Delphi y grupos focales. El fin era articular una discusión en profundidad para poder llegar a la primera propuesta de capacidades en cada país. Para ello, era necesaria una prevalencia de metodología cualitativa, a fin de lograr una primera aproximación al objeto de estudio (un marco de capacidades). Y, dado que se trata de una realidad conceptual nueva para la disciplina, se consideró que un panel de expertos Delphi y grupos focales eran los dos mejores métodos para tal fin.

La investigación tuvo dos fases en España y Argentina 2016 y 2017, que se resumen a continuación, y más adelante se especifican los criterios de muestras por país. En una primera fase, con el panel de expertos de Delphi se pretendió llegar a la definición de un marco inicial de capacidades que, a su juicio, definiera el potencial de la profesión y sus profesionales. Este método ofrece oportunidades para consultar a expertos sobre una realidad con desafíos conceptuales; y así ha sido aplicado en la historia reciente de la disciplina (cfr. Ruler, Vercic, Bütschi y Flodin, 2009; Wakefield y Watson, 2014; Watson, 2008), al tiempo que brinda una oportunidad analítica para proponer contenido sobre una temática incipiente (Linstone y Turoff, 1975), construyendo un consenso entre la variedad de opiniones. El panel Delphi posibilitó en cada país un consenso fiable de un grupo anónimo de expertos mediante la realización de cuestionarios intensivos con retroalimentación controlada.

El método Delphi se ejecutó en tres rondas, como marca su naturaleza metodológica, con cuestionarios abiertos y cerrados. Los investigadores recogieron el bruto de la información, la analizaron y realizaron una síntesis. Posteriormente, en la(s) siguiente(s) ronda(s) se presentó a los panelistas los hallazgos compilados de forma anónima, pidiendo una valoración de sus posiciones iniciales respecto al conjunto de respuestas y pudiendo aportar feedback adicional (Delbecq, Van de Ven y Gustafson, 1975).

En la segunda parte cualitativa, y sobre la base de los marcos de capacidades obtenidos en los paneles Delphi, se organizaron grupos de discusión para que los expertos académicos y profesionales, con igual criterio de muestra, debatieran si el marco de capacidades propuesto por los expertos del panel tendría un valor práctico para el desarrollo de la profesión en todos sus niveles: individual, nacional y globalmente.

Los grupos focales de discusión son una metodología especialmente adecuada para estudios que tienen por objeto confirmar una información, o para realidades conceptuales incipientes (Barbour, 2008). Éstos se ejecutaron siguiendo tres fases estructuradas (Stewart y Shamdasani, 1990). En primer lugar, los moderadores explicaron el propósito de la investigación, así como las principales cuestiones que pretendían abordarse. En segundo lugar, se facilitó un cuestionario individual a cada participante, al objeto de recoger sus valoraciones individuales, antes del comienzo de la discusión. Por último, la discusión se organizó en torno a tres cuestiones clave, adaptadas según se tratara de un grupo focal de académicos, empleadores o profesionales. Las tres preguntas clave fueron cuestionar (a) si consideraban que el marco de capacidades del panel Delphi era relevante y si realizarían alguna enmienda o modificación; (b) si el marco supondría una referencia útil para la profesión, la academia y las asociaciones profesionales y (c) si el marco resultaría de interés y aplicación práctica para apoyar el desarrollo profesional individual y de equipos, así como el diseño curricular de educación superior. 
Siguiendo a Krueger (1998), los investigadores: a) revisaron la información obtenida en los focus groups, utilizando los apuntes del moderador y las transcripciones de las grabaciones de audio; b) identificaron los hallazgos más trascendentes y los agruparon según las categorías temáticas; c) analizaron las posibles diferencias por variables muestrales y los aspectos de confluencia y disensos; y, d) evaluaron la fortaleza de los resultados ilustrándolos con citas textuales.

En este artículo, los hallazgos relativos a los grupos focales se circunscriben a los resultados de la discusión y no a las respuestas de los cuestionarios individuales, por razones de síntesis y por preferir ofrecer qué puntos de discusión fueron relevantes en la interacción. Antes, para comprender los hallazgos, se ofrece una breve descripción del contexto de cada país, para dar cuenta de los criterios de selección de las muestras, así como el trabajo de campo.

\subsection{España: contexto profesional y muestra del estudio}

En España, la profesión en general se caracteriza por su juventud, y ha estado muy marcada por la evolución política, económica y social del siglo XX (Gutiérrez-García y Rodríguez Salcedo, 2009; Moreno, 2004; Rodríguez Salcedo, 2008). Para comprender mejor el debate suscitado en el panel Delphi y los grupos focales, es pertinente resaltar varias pinceladas sobre el contexto.

Cómo se va conformando una profesión se comprende por su desarrollo histórico, y al respecto, Rodríguez-Salcedo destaca que España es un ejemplo de cómo emergió en un régimen no democrático, desafiando la historiografía dominante anglosajona (2015, p. 213). La profesión nació en el siglo XX y, como afirman Rodríguez-Salcedo y Watson "el contexto político y social en el final de la dictadura de Franco dio forma al pionero desarrollo de las relaciones públicas en España" (2017, p. 376). Una de las primeras agencias profesionales documentadas fue fundada en 1960, SAE de Relaciones Públicas (Montero et al., 2010), gracias a las reformas económicas y políticas impulsadas por el régimen de Franco, con una mayor apertura hacia el emprendimiento empresarial, incluyendo las consultoras (Rodríguez-Salcedo, 2015, p. 214).

Seguido del incipiente desarrollo profesional de los 60, especialmente de consultoría, se promovió el primer movimiento asociativo: en 1961 la Asociación Técnica de Relaciones Públicas y en 1965 el Centro Español de Relaciones Públicas (ambas extintas). Esta primera oleada asociativa sólo ha tenido parangón en la historia a finales del siglo XX: en 1992 nace la Asociación de Directivos de Comunicación-DIRCOM-, y antes, en 1991, la Asociación de Empresas Consultoras en Relaciones Públicas y Comunicación -ADECEC- (activas actualmente).

El desarrollo profesional desde el siglo XX no ha ido acompañado, sin embargo, de igual crecimiento en la oferta curricular universitaria. En 1968 se fundó la primera iniciativa: la Escuela Superior de Relaciones Públicas (ESERP). Y no fue hasta $1971^{3}$ cuando el Ministerio de Educación reguló los estudios superiores, dando paso a la fundación de las entonces Facultades de Ciencias de la Información, otorgando, por primera vez, categoría universitaria a la disciplina, y reconociendo títulos de pregrado como Periodismo o Publicidad y Relaciones Públicas. Fue en el curso 1971/1972 cuando por primera vez se impartieron programas especializados, denominados Publicidad y Relaciones Públicas, en las universidades Autónoma de Barcelona y Complutense de Madrid. Una Orden Ministerial de 1974 reconocía provisionalmente el currículo formativo de las Facultades ${ }^{4}$; pero no fue

\footnotetext{
${ }^{3}$ Decreto 2070/1971, de 13 de agosto, por el que se regulan los estudios de Periodismo y demás medios de comunicación social en la Universidad. Boletín Oficial del Estado -BOE-A-1971-1180. Consultado en: https://www.boe. es/boe/dias/1971/09/14/pdfs/A14944-14945.pdf.

4 "Orden de 14 de septiembre de 1974 por la que se aprueba el plan provisional de estudios correspondientes a las Facultades de Ciencias de la Información”. Número de referencia: BOE-A-1974-1560. Consultado en: https:// www.boe.es/boe/dias/1974/09/24/pdfs/A19534-19535.pdf.
} 
hasta los 90 cuando comenzaron de modo generalizado 5 . A pesar de ello, según un reciente análisis, la profesión no cuenta con una formación universitaria estandarizada en grados y posgrados, siendo la situación actual compleja y diversa (Saavedra \& Perlado, 2017).

La breve reseña histórica es muy relevante para comprender por qué ha emergido un marco de capacidades que incluye una recomendación unánime de profesionales y académicos sobre la pertinencia de seguir indagando en la naturaleza de la profesión.

\section{Muestra en España: panel Delphi y grupos de discusión}

La selección de la muestra del panel Delphi y los grupos focales ha tenido en cuenta tres criterios: académicos con amplia experiencia docente e investigadora, y profesionales con responsabilidades en equipos y contratación. Como se observa en las tablas, se ha buscado intencionalmente una representatividad mayor de los profesionales, al ser un proyecto centrado en la reflexión sobre el potencial de la profesión.

No resulta posible saber con precisión cuál es el universo de estudio español, porque no existe un registro oficial que dictamine cifras sobre los profesionales, ni exactas ni aproximadas, tampoco en las estadísticas oficiales sobre profesiones de la Secretaría de Estado de Empleo del Gobierno de España. Por su parte, el Anuario de la Comunicación, publicación de referencia editada por la Asociación DIRCOM, contiene una base de datos con más de 3.000 profesionales de consultorías y departamentos de comunicación de entidades (públicas, privadas y tercer sector). Por otro lado, tampoco resulta fácil determinar el universo de académicos. Existen alrededor de 115.000 docentes en España (Ministerio de Educación, 2017) pero no se especifica el número que investiga en las áreas de relaciones públicas y/o comunicación estratégica. Se estima que la disciplina de comunicación cuenta con 162 grados universitarios, y muestra una gran dispersión temática, sumando Periodismo, Audiovisual, Publicidad o Relaciones Públicas (Saavedra y Perlado, 2017).

A fin de seleccionar el panel de expertos Delphi, se invitó a participar a 21 personas que cumplían los criterios mencionados y aceptaron 17. Los perfiles detallados del panel en las tres rondas se muestran en las tablas 1 y 2 .

Tabla 1. Número de participantes en las rondas del panel Delphi

\begin{tabular}{|l|c|c|c|c|c|}
\hline Participantes & Invitados & Aceptación & $\mathbf{1}^{\text {a }}$ ronda & $2^{\text {a }}$ ronda & $3^{\text {a }}$ ronda \\
\hline Profesionales senior & 11 & 8 & 8 & 6 & 6 \\
\hline Empleadores & 5 & 5 & 5 & 5 & 5 \\
\hline Académicos & 5 & 4 & 4 & 4 & 3 \\
\hline Total & 21 & 17 & 17 & 15 & 14 \\
\hline
\end{tabular}

Fuente: elaboración propia (trabajo de campo: entre febrero y junio de 2017)

\footnotetext{
5 "Real Decreto 1383/1991, de 30 de agosto, por el que se establece el título universitario oficial de Licenciado en Publicidad y Relaciones Públicas y las directrices generales propias de los planes de estudio conducentes a la obtención de aquél". Número de referencia: BOE-A-1991-24112. Consultado en: https://www.boe.es/boe/ dias/1991/09/30/pdfs/A31775-31777.pdf.
} 
Tabla 2. Perfil detallado de la muestra (total n.17)

\begin{tabular}{|c|c|c|c|}
\hline Perfil profesional & Frecuencia & Edad & Frecuencia \\
\hline Profesionales senior & 8 & $40-49$ & 9 \\
\hline Empleadores & 5 & $50-59$ & 4 \\
\hline Académicos & 4 & 60 o más & 4 \\
\hline Género & Frecuencia & Ciudad & Frecuencia \\
\hline Hombres & 11 & Madrid & 11 \\
\hline \multirow[t]{2}{*}{ Mujeres } & 6 & Barcelona & 2 \\
\hline & & Otros & 4 \\
\hline Educación & Frecuencia & Ubicación* (n.13) & Frecuencia \\
\hline Sin educación superior & 1 & In-house & 5 \\
\hline Carrera universitaria & 7 & Consultoría & 6 \\
\hline Master & 3 & Otros (freelance) & 2 \\
\hline Doctorado & 6 & & \\
\hline Tipo de organización* (n.13) & Frecuencia & & \\
\hline Privada & 2 & & \\
\hline Pública & 2 & & \\
\hline Administración pública & 1 & & \\
\hline Consultoría & 7 & & \\
\hline Tercer sector & 1 & & \\
\hline
\end{tabular}

*Nota: sólo profesionales senior y empleadores.

Fuente: elaboración propia

Para los grupos focales, se seleccionó la muestra con idénticos criterios que en el panel Delphi. Un total de 54 personas (14 académicos y 40 profesionales) participaron en siete grupos de discusión organizados en cuatro ciudades españolas (Madrid, Barcelona, Valladolid, y Valencia), a fin de asegurar la representatividad de sectores, organizaciones y tamaños. En las tablas 3 y 4 se detalla la muestra. La formación universitaria es una pregunta con respuesta múltiple.

Tabla 3. Perfil de los académicos (n. 14)

\begin{tabular}{|c|c|c|c|c|c|}
\hline Categoría académica* & Frecuencia & Antigüedad o experiencia & Frecuencia & Género & Frecuencia \\
\hline Catedrático & 3 & 11-15 años & 2 & Masculino & 8 \\
\hline Prof. Titular & 4 & $16-20$ años & 4 & Femenino & 6 \\
\hline Prof. Contratado Doctor & 4 & 21 años o más & 8 & & \\
\hline Prof. Asociado & 2 & & & & \\
\hline Edad & Frecuencia & Formación universitaria* & Frecuencia & & \\
\hline $35-44$ & 4 & Lcdo. Periodismo & 2 & & \\
\hline $45-54$ & 6 & $\begin{array}{l}\text { Lcdo. Publicidad y Relaciones } \\
\text { Públicas }\end{array}$ & 4 & & \\
\hline $55-64$ & 2 & Master en Comunicación & 3 & & \\
\hline Más de 65 & 2 & Doctorado & 11 & & \\
\hline
\end{tabular}

Fuente: elaboración propia (trabajo de campo: noviembre y diciembre de 2017) 
Tabla 4. Perfil de los profesionales (n. 40)

\begin{tabular}{|c|c|c|c|c|c|}
\hline Sector & Frecuencia & Experiencia profesional & Frecuencia & Edad & Frecuencia \\
\hline Privado & 31 & $11-15$ años & 7 & $35-44$ & 5 \\
\hline Educativo & 3 & 16-20 años & 8 & $45-54$ & 25 \\
\hline Público & 2 & 21 años o más & 25 & $55-64$ & 8 \\
\hline Asociaciones & 4 & & & Más de 65 & 2 \\
\hline Actividad profesional & Frecuencia & Género & Frecuencia & $\begin{array}{l}\text { Miembro de } \\
\text { asociaciones } \\
\text { profesionales }\end{array}$ & Frecuencia \\
\hline In-House (director) & 27 & Hombres & 23 & Sí & 38 \\
\hline Consultoría & 13 & Mujeres & 17 & No & 2 \\
\hline Formación universitaria* & Frecuencia & $\begin{array}{l}\text { Salarios (anual, en } \\
\text { euros) }\end{array}$ & Frecuencia & Salarios (cont.) & Frecuencia \\
\hline Sin estudios universitarios & 1 & $25.000-35.000$ & 2 & $95.001-105.000$ & 2 \\
\hline Periodismo & 25 & $35.001-45.000$ & 5 & Más de 105.000 & 15 \\
\hline $\begin{array}{l}\text { Publicidad y Relaciones } \\
\text { Públicas }\end{array}$ & 3 & $45.001-55.000$ & 1 & $\begin{array}{l}\text { Prefiere no } \\
\text { responder }\end{array}$ & 9 \\
\hline Master en Comunicación & 7 & $55.001-65.000$ & 1 & & \\
\hline MBA & 4 & $65.001-75.000$ & 3 & & \\
\hline $\begin{array}{l}\text { Grados en otras disciplinas } \\
\text { (Económicas, Psicología, } \\
\text { Derecho, Química, Historia } \\
\text { \& Artes) }\end{array}$ & 12 & $75.001-85.000$ & 2 & & \\
\hline Doctorado & 5 & $85.001-95.000$ & 0 & & \\
\hline
\end{tabular}

Fuente: elaboración propia (trabajo de campo: noviembre y diciembre de 2017)

\subsection{Argentina: contexto profesional y muestra}

El origen de la profesión en Argentina se remonta a fines de 1940 y principios de 1950, con las primeras prácticas profesionales sistemáticas ocurridas en las filiales de algunas empresas multinacionales que replicaban modelos exitosos de sus países de origen (Ferrari y França, 2011; Carbone y Montaner, 2014).

La creación del primer colectivo profesional, consecuencia natural de una práctica en evolución, ocurrió en 1958, con el establecimiento de la Asociación Argentina de Relaciones Públicas, que años más tarde se convertiría en el Consejo Profesional de Relaciones Públicas de la República Argentina (CPRPRA), en la actualidad la principal entidad representativa de la profesión y miembro de la federación Global Alliance.

En aquel contexto, la formalización de propuestas de educación se dio de modo acelerado, con la creación en 1964 de la primera licenciatura (pregrado) en relaciones públicas -primera en el mundo de habla hispana- en la Universidad Argentina de la Empresa (UADE). En 2018 son 20 las casas de altos estudios en todo el país que tienen la carrera en el pregrado, con un total de 8516 estudiantes $^{6}$.

La práctica parece haber llegado a un signo de maduración en las dos últimas décadas (Ferrari y França, 2011), con una instalación mayoritaria en departamentos de organizaciones medianas y grandes, tanto en el sector estatal, privado y social, y una oferta muy nutrida de servicios por agencias o consultoras -sobre todo en el área metropolitana de Buenos Aires-, aunque con un área de va-

\footnotetext{
${ }^{6}$ Información oficial de la Secretaría de Políticas Universitarias de Presidencia de la Nación. Consultado en http:// estadisticasuniversitarias.me.gov.ar/\#/seccion/1
} 
cancia importante aún en lo que respecta a la investigación académica (Álvarez Nobell et al., 2016).

\section{Muestra: panel Delphi y grupos de discusión}

A grandes rasgos, los criterios de selección de la muestra, tanto para el Delphi como para los grupos de discusión, giraron en torno a que los profesionales y académicos fueran líderes experimentados y que los empleadores dirigieran o tuvieran a su cargo el área de Recursos Humanos de las consultoras especializadas más importantes del país en cantidad de personas contratadas.

Como se evidencia en las tablas, la representatividad otorgada a cada uno fue similar, aunque vale consignar que en el caso de los empleadores su condición podía solaparse con la de los profesionales, al ser casi todos los máximos responsables de sus consultoras. De este modo, se adecuó la muestra a la intención del proyecto, es decir, centrar su interés en la reflexión sobre el potencial de la profesión.

Al igual que en España, en Argentina tampoco resulta posible saber con precisión el universo de estudio. No existe un registro oficial que brinde cifras acerca de la cantidad de profesionales o académicos especializados. Una mera y muy preliminar estimación, basada en la cantidad de estudiantes que cursan la carrera en el pregrado y el nivel relativo de desarrollo de la profesión en países con similares estadios y estadísticas oficiales, arroja una cifra de entre 15.000 y 20.000 profesionales en total (Fawkes et al., 2018).

En el proceso de selección del panel de expertos, a fin de conseguir una cantidad pertinente, se invitó a participar a 19 personas, seis profesionales, seis académicos y siete empleadores.

Los criterios de selección para los académicos fueron: a) directores o coordinadores de carreras de universidades validadas por el CPRPRA, b) docentes con experiencia en investigación, c) docentes con participación en debates sobre la profesión y sus tendencias. Los profesionales senior, en tanto, debían ser: a) miembros del CPRPRA, en cualquier sector de actividad, b) interesados en la participación en debates, o autores de materiales sobre la evolución profesional. Finalmente, los empleadores debían: a) ser reconocidos como líderes en sus ámbitos, b) evidenciar su interés por las comunicaciones, habiendo participado en conferencias, c) ser los responsables finales de reclutar personas para equipos de 20 o más integrantes. En todos los casos, los panelistas no necesitaron cumplir todos los criterios para cada perfil y, además, se trató de abarcar un grupo de personas geográfica, sectorial y etariamente diversas, del mismo modo que en materia de género, aunque, como se ha mencionado, sin la intención de ser representativo de la profesión. Los perfiles detallados del panel para las tres rondas se muestran en las tablas 5 y 6 .

Tabla 5. Número de participantes en las rondas del Delphi

\begin{tabular}{|l|c|c|c|c|c|} 
& Invitados & Aceptación & $1^{\text {a }}$ ronda & $2^{\text {a }}$ ronda & $3^{\text {a }}$ ronda \\
\hline Profesionales senior & 6 & 6 & 5 & 5 & 4 \\
\hline Empleadores & 7 & 7 & 3 & 3 & 3 \\
\hline Académicos & 6 & 6 & 6 & 6 & 6 \\
\hline Total & 19 & 19 & 14 & 14 & 12 \\
\hline
\end{tabular}

Fuente: elaboración propia (trabajo de campo: entre octubre de 2016 y enero de 2017) 
Tabla 6. Perfil de la muestra (total n.19)

\begin{tabular}{|c|c|c|c|}
\hline Perfil profesional & Frecuencia & Edad & Frecuencia \\
\hline Profesionales senior & 6 & 30-39 & 2 \\
\hline Empleadores & 7 & $40-49$ & 10 \\
\hline Académicos & 6 & $50-59$ & 7 \\
\hline Género & Frecuencia & Ciudad & Frecuencia \\
\hline Hombres & 12 & Buenos Aires & 16 \\
\hline Mujeres & 7 & $\begin{array}{l}\text { Otros (Córdoba, Rosario, Men- } \\
\text { doza) }\end{array}$ & 3 \\
\hline Educación & Frecuencia & Ubicación* (n.13) & Frecuencia \\
\hline Carrera universitaria & 7 & In-house & 6 \\
\hline Maestría & 8 & Consultoría & 7 \\
\hline Doctorado & 4 & & \\
\hline Tipo de organización* (n.13) & Frecuencia & & \\
\hline Privada & 2 & & \\
\hline Pública & 2 & & \\
\hline Administración pública & 1 & & \\
\hline Consultoría & 7 & & \\
\hline Tercer sector & 1 & & \\
\hline
\end{tabular}

${ }^{\star}$ Nota: sólo profesionales y empleadores

Fuente: elaboración propia

Para los grupos focales, se seleccionó la muestra con mismos perfiles que el panel Delphi. Un total de 27 personas participaron en tres grupos de discusión, que se complementaron con entrevistas a tres personas (dos profesionales y una académica) de otras ciudades del país, provenientes de organizaciones de diferentes sectores y tamaños. En las tablas 7, 8 y 9 se detalla la muestra. La formación universitaria es una pregunta con respuesta múltiple.

Tabla 7. Perfil de los académicos (n.11)

\begin{tabular}{|c|c|c|c|c|c|}
\hline Categoría académica & Frecuencia & Antigüedad o experiencia & Frecuencia & Género & Frecuencia \\
\hline Prof. titular & 6 & 11-15 años & 3 & Masculino & 3 \\
\hline \multirow[t]{2}{*}{ Prof. asociado } & 5 & 16-20 años & 4 & Femenino & 8 \\
\hline & & 21 años o más & 4 & & \\
\hline Edad & Frecuencia & Formación universitaria* & Frecuencia & & \\
\hline $35-44$ & 4 & Lic. Periodismo o Comunicación & 3 & & \\
\hline $45-54$ & 5 & Lic. Relaciones Públicas & 7 & & \\
\hline \multirow[t]{2}{*}{$55-64$} & 2 & Maestría & 7 & & \\
\hline & 2 & Doctorado & 2 & & \\
\hline
\end{tabular}

Fuente: elaboración propia (trabajo de campo: septiembre y octubre de 2017) 
Tabla 8. Perfil de los profesionales (n.11)

\begin{tabular}{|c|c|c|c|c|c|}
\hline Sector & Frecuencia & Experiencia profesional & Frecuencia & Edad & Frecuencia \\
\hline Privado & 6 & 11-15 años & 3 & $35-44$ & 5 \\
\hline Administración Pública & 4 & $16-20$ años & 4 & $45-54$ & 5 \\
\hline Social & 1 & 21 años o más & 4 & $55-64$ & 1 \\
\hline Actividad profesional & Frecuencia & Género & Frecuencia & $\begin{array}{l}\text { Miembro de } \\
\text { asociaciones } \\
\text { profesionales }\end{array}$ & Frecuencia \\
\hline In-House (director) & 9 & Hombres & 8 & Sí & 10 \\
\hline Consultoría & 2 & Mujeres & 3 & No & 1 \\
\hline Formación universitaria* & Frecuencia & & & & \\
\hline Sin estudios universitarios & 0 & & & & \\
\hline Periodismo o Comunicación & 5 & & & & \\
\hline Relaciones Públicas & 4 & & & & \\
\hline Maestría & 6 & & & & \\
\hline \begin{tabular}{|l|} 
Grados en otras disciplinas \\
(ciencias políticas, ingeniería)
\end{tabular} & 2 & & & & \\
\hline Doctorado & 0 & & & & \\
\hline
\end{tabular}

Fuente: elaboración propia (trabajo de campo: septiembre y octubre de 2017)

Tabla 9. Perfil de los empleadores (n.5)

\begin{tabular}{|l|c|l|c|}
\hline Género & Frecuencia & Antigüedad o experiencia & Frecuencia \\
\hline Masculino & 1 & $11-15$ años & 2 \\
\hline Femenino & 4 & $16-20$ años & 2 \\
\hline & & Más de 21 años & 1 \\
\hline Edad & Frecuencia & Formación universitaria* & Frecuencia \\
\hline $35-44$ & 2 & Lic. Periodismo o Comunicación & 1 \\
\hline $45-54$ & 2 & Lic. Relaciones Públicas & 1 \\
\hline $55-64$ & 1 & Lic. RR. HH. & 2 \\
\hline & & Maestría & 4 \\
\hline
\end{tabular}

Fuente: elaboración propia (trabajo de campo: septiembre y octubre de 2017)

\section{Hallazgos españoles y argentinos}

\section{España}

El resultado final de la investigación en España ha generado la propuesta de un marco consensuado de 9 capacidades. Se ha llegado a él gracias a las tres rondas con los 14 expertos -académicos y profesionales- del panel Delphi, que dio como resultado un listado preliminar de 14 capacidades; posteriormente sometido a la discusión y valoración por 54 expertos agrupados en los siete grupos de discusión.

Las tres rondas se sucedieron entre febrero y junio de 2017. La primera constó de preguntas abiertas, incluyendo una hoja explicativa del concepto de capacidad; y se pidió a los expertos que aportaran individualmente, a su juicio, una lista de las 10 más importantes para el futuro de la profesión, según su naturaleza estratégica, operativa y genérica. El resultado de la primera 
ronda fueron más de 50 capacidades que fueron interpretadas y codificadas en dos fases: primero, agrupándolas según las categorías mencionadas (estratégicas, operativas y genéricas). Segundo, se analizaron solapamientos y repeticiones, a fin de realizar una síntesis y un listado inicial que fue sometido al análisis en las dos siguientes rondas. En dichas rondas se pidió a los expertos que valoraran el listado resultante en una escala (1. Nada importante a 7. Extremadamente importante), arrojando un alto consenso; lo cual mostró la correcta síntesis, así como la confluencia de opiniones. En la tercera ronda se solicitó elaborar un ranking del listado, del 1 al 14; pero no hubo consenso y generó polarización sobre el orden de importancia (véase detalles en Gutiérrez-García \& Recalde, 2018).

Tabla 10. Listado de capacidades propuesta por el panel Delphi. España

\begin{tabular}{|l|}
\hline A. Diseñar planes estratégicos de comunicación, alineados con los objetivos corporativos \\
\hline B. Apoyar la gestión de intangibles como cultura, propósitos y valores \\
\hline C. Analizar e interpretar el entorno (inteligencia social) para identificar oportunidades y mitigar riesgos \\
\hline D. Contribuir a gestionar relaciones con stakeholders \\
\hline E. Asesorar en temas de comunicación y cohesionar a la dirección \\
\hline $\begin{array}{l}\text { F. Alinear equipos y departamentos, creando sinergias internas y externas, contribuyendo a generar oportuni- } \\
\text { dades y evitar conflictos }\end{array}$ \\
\hline G. Capacidad para tomar decisiones, resolución de problemas y orientación a la acción \\
\hline H. Construir una narrativa organizativa que ayude a la relación con stakeholders y mantener la consistencia \\
\hline I. Trasladar conceptos e información compleja a mensajes y acciones concretas \\
\hline J. Generar contenidos en medios tradicionales y digitales \\
\hline K. Medir, monitorear y evaluar \\
\hline L. Liderar y dirigir equipos multidisciplinares y multiculturales \\
\hline M. Capacidad creativa para proponer soluciones innovadoras y promover transformaciones \\
\hline N. Gestionar competencias y actitudes profesionales \\
\hline
\end{tabular}

Fuente: elaboración propia.

La lista resultante fue sometida a discusión en los siete grupos focales, a fin de lograr, como se ha señalado, dar respuesta a las tres preguntas explicadas en el epígrafe anterior. En síntesis, se puede afirmar que, en los siete grupos de discusión, tanto académicos como profesionales, se logró un gran consenso sobre la pertinencia y utilidad de definir un marco de capacidades. A su juicio, facilitaría tener una mirada enfocada a la esencia de la profesión, especialmente tras décadas de debates conceptuales y variedad lexicológica. Al respecto, un participante señalaba de modo ilustrador:

Necesitamos criterios más claros, estandarización; porque actualmente tenemos muy diferentes interpretaciones sobre qué debería ser la Comunicación, no hay consenso en absoluto. Y esta situación no debería durar si queremos ser reconocidos como una función estratégica en las organizaciones. Por ello, un marco de capacidades podría ayudarnos a presentarnos mejor a nosotros mismos (Profesional senior, FG\#4, 30 noviembre 2017).

A continuación, se sintetizan los hallazgos sobre las tres preguntas clave:

\section{¿Modificarían o enmendarían el marco propuesto por el panel Delphi?}

Cuando se solicitó a los participantes que hicieran comentarios y enmiendas a la lista preliminar propuesta por el panel Delphi, se alcanzó un amplio consenso en todas las sesiones. Tanto académicos como profesionales reconocieron que dicho listado proporcionaba respuestas a los grandes 
desafíos. Todos, no obstante, acordaron abreviar y sintetizar el listado; aunque en las discusiones emergieron nuevas capacidades y sub-capacidades.

Los participantes, tanto académicos como profesionales, llegaron al consenso sobre la necesidad de reflexionar sobre los desafíos futuros de la profesión. Entre ellos, el liderazgo, concebido desde una perspectiva estratégica, una capacidad ampliamente subrayada. Con todo, ser un asesor de confianza y dirigir su liderazgo, como señalaba un profesional, "tiene mucho que ver con la medición y con los intangibles (...) y creo que no está lo suficientemente desarrollado" (Profesional senior, FG\#6, 1 diciembre 2017).

Por otro lado, académicos y profesionales coincidieron en aconsejar categorizar las capacidades según niveles profesionales y experiencia. Sin embargo, no todo fueron consensos. Entre las capacidades que provocaron una alta polarización en el debate se destaca la $\mathrm{N}$ (referida a habilidades y actitudes personales y genéricas). Tampoco hubo un acuerdo respecto a la capacidad M (capacidad creativa para proponer soluciones innovadoras y promover transformaciones). Por último, destacaron que la ética debería ser expresada de forma más explícita en el marco resultante:

Ser un poco la conciencia de la organización, el ver un poco lo que es apropiado, correcto e incorrecto. $\mathrm{Y}$ otra, que es un poco complementaria, ser el sensor, de la relación con los grupos de interés y de ahí el proceso de la escucha (Profesional senior, FG\#5, 30 noviembre 2017).

¿Qué utilidad tendría un marco de capacidades para profesionales, académicos y asociaciones profesionales?

Ante la histórica confusión terminológica y la juventud de la profesión, el marco ayudaría, de acuerdo con los expertos, a que se reconociera la misión de la profesión. Los consultores acentuaron en varios grupos de discusión que un marco consensuado es clave para su sector; idea en la que coincidieron muchos directores de comunicación pues, al mismo tiempo, sería un indicador de la madurez de la profesión.

La necesidad de programas de capacitación profesional individual fue un tema que se repitió constantemente, junto al reconocimiento de los profesionales de un papel más proactivo que debería jugar la universidad. Si bien, los participantes no alcanzaron un consenso sobre qué tipo de programas serían los más apropiados.

¿Cómo puede un marco de este tipo apoyar el desarrollo profesional individual, nacional y global?

Todos los grupos abrieron el debate afirmando que la profesión debe entenderse dentro de las organizaciones y la sociedad en general. Al respecto, un profesional con experiencia en tres países europeos, manifiesta la falta de una definición clara de la profesión: "Veo este mismo problema en otros países (...) Cuando estás hablando de unas capacidades específicas, a lo mejor nunca han sido conscientes de que estos son elementos que se necesitan para ser un buen comunicador" (Profesional senior, FG\#5, 30 noviembre 2017). Una posible explicación es que las especificidades de esta profesión son difíciles de determinar comparándola con otras (finanzas, operaciones, legal, etc.), tal y como ya se destacó en las mencionadas investigaciones pioneras de Broom y Smith.

Por otro lado, los profesionales y académicos mostraron su acuerdo con que un marco de capacidades ayudaría a establecer más sistemáticamente los programas formativos y de desarrollo profesional permanente, con la mirada puesta en cómo capacitar a los equipos para el futuro.

\section{$\underline{\text { Marco final de capacidades }}$}

La lista mostrada en la tabla 11 es resultado del análisis de la discusión de los siete grupos en España, junto al análisis de los comentarios individuales que cada participante escribió previo a la discusión de los grupos. 
Tabla 11. Marco final de capacidades. España

\begin{tabular}{|l|}
\hline $\begin{array}{l}\text { A. Diseñar planes y estrategias alineados con los objetivos orga- } \\
\text { nizativos }\end{array}$ \\
\hline B. Apoyar y mejorar la gestión de intangibles \\
\hline C. Ser un consejero y asesor confiable, liderando cambios \\
\hline D. Contribuir a gestionar las relaciones con los grupos de interés \\
\hline E. Ser una conciencia crítica y ética en la organización \\
\hline F. Gestionar múltiples lenguajes ante los desafíos digitales \\
\hline G. Ser creativo e innovador \\
\hline H. Dominar las diferentes herramientas y tácticas comunicativas \\
\hline I. Tener conocimientos de gestión y aspectos financieros \\
\hline
\end{tabular}

Fuente: elaboración propia.

\section{Argentina}

El resultado final de la investigación en Argentina generó un marco consensuado de 11 capacidades. Se llegó a él gracias a las tres rondas Delphi, mediante un listado preliminar de 13 capacidades que, posteriormente, y luego de una etapa masiva administrada a través de una encuesta, fue sometido a la discusión y valoración de 27 expertos en tres grupos focales de discusión.

De igual manera que en España, y en los otros siete países que participaron del estudio, el Delphi se ejecutó en tres rondas, en el caso argentino entre octubre de 2016 y enero de 2017, con cuestionarios tanto abiertos como cerrados, presentando a los expertos los hallazgos sucesivos de forma individual y anónima y solicitando sus opiniones y feedback adicionales.

El primer cuestionario, enviado por correo electrónico de forma individual, comprendió una breve introducción al estudio y la invitación a definir un conjunto de 10 capacidades básicas. También incluyó una desambiguación del término capacidades y las definiciones para agrupar entre capacidades de comunicación estratégica, operativa y genéricas.

A partir de la recepción de los resultados, se los agrupó en relación con cada tipo de capacidad y, a partir de las similitudes, en un código conceptual. Así se clasificaron 67 capacidades estratégicas, 67 operativas y 68 genéricas, estandarizándose las respuestas y logrando una codificación común consistente en 17 estratégicas, 16 operativas y 13 genéricas. Se le asignó un valor correspondiente a un voto por cada participante que lo indicara y, a partir de esta evaluación, se llegó a una lista de las 14 capacidades más votadas para ser enviadas en la segunda ronda.

En ella, se invitó a los participantes a clasificar las capacidades en una escala (de 1. Nada importante a 7. Extremadamente importante). Además de trabajar sobre las capacidades más repetidas en el primer cuestionario, los participantes tuvieron la libertad de añadir otras si las creían de importancia capital y no figuraban en la lista. Durante el análisis de los datos se consideraron los valores dados por los participantes y la cantidad de repeticiones de cada valoración. En la tercera y última ronda del Delphi, se solicitó a los panelistas que seleccionaran las 10 capacidades más importantes, siendo 1 la más favorita y 10 la menos. Durante el análisis de los resultados, se tabularon las capacidades identificadas con letras del alfabeto en una tabla de entrada doble en la que se otorgó el valor de un voto a cada capacidad según el orden de preferencia otorgado por cada panelista. Esta representación gráfica permitió identificar el número de veces que se ligó una capacidad con la misma valoración, eliminando una en función de la casi nula puntuación obtenida. El listado resultante de 13 capacidades, en orden decreciente de importancia relativa, se puede ver en la tabla 12. 
Tabla 12. Listado de capacidades surgida del panel Delphi. Argentina

\begin{tabular}{|l|}
\hline $\begin{array}{l}\text { A. Comprender el negocio/actividad de la organización/cliente y formular estrategias de relacionamiento y co- } \\
\text { municación que les den soporte }\end{array}$ \\
\hline B. Contribuir al logro de los objetivos de la organización (objetivos de negocio, sociales o de política pública) \\
\hline C. Comprender el entorno sociopolítico-económico de la organización/cliente \\
\hline D. Elaborar contenidos, informes y reportes profesionales \\
\hline E. Conocer y saber usar los canales y plataformas de comunicación \\
\hline F. Comprender a los públicos y generar vínculos a largo plazo basados en la comunicación efectiva y el con- \\
senso \\
\hline G. Reconocer, gestionar y comunicar la identidad de la organización sin perder la propia personalidad \\
\hline H. Conducirse sobre la base de principios éticos, valores de inclusión social y diversidad \\
\hline I. Capacitarse de manera constante a lo largo de toda la carrera \\
\hline J. Establecer métricas confiables para medir el progreso de los objetivos \\
\hline K. Ser flexible y adaptarse a los cambios \\
\hline L. Saber negociar, relacionarse con empatía y de modo persuasivo, aún en momentos críticos \\
\hline M. Realizar tareas múltiples en el marco de equipos multidisciplinarios \\
\hline
\end{tabular}

Fuente: elaboración propia.

En cuanto a la fase de los tres grupos focales de discusión, se desarrolló entre septiembre y octubre de 2017, uno para cada perfil consignado: profesionales, académicos y empleadores. La pregunta de investigación de esta fase final residió en el valor práctico del marco de capacidades profesionales resultante, tanto para la profesión como para la academia.

Durante cada sesión se presentó y distribuyó una hoja con preguntas demográficas simples mientras se presentaba el grupo focal, con un especial cuidado en explicar las diferencias entre capacidad y competencia.

En los grupos focales realizados se discutió la existencia de solapamientos entre algunas de las capacidades y se destacó la importancia de la capacidad de ser flexible, ya que "en países como el nuestro, con una realidad política y económica tan cambiante, los profesionales tienen que tener desarrollada la capacidad de adaptarse a los cambios" (profesional senior, FG\#2, 14 de septiembre de 2017).

Los principios éticos también fueron puestos en discusión, pero por el hecho de que "la capacidad de conducirse sobre la base de principios éticos, valores de inclusión social y diversidad es transversal a cualquier tipo de profesión. No es que no nos parece relevante, sino que la consideramos como algo común con todas las profesiones" (empleadora, FG\#1, 4 de septiembre de 2017).

A su vez, se debatió y acordó la necesidad de un desarrollo profesional continuo y de promover la capacidad de negociación. También se realzó en las tres reuniones, aunque particularmente en la de académicos y profesionales, que la contribución al logro de los objetivos de la organización debía incluir objetivos comerciales, pero también sociales.

Sobre el valor práctico del marco de capacidades resultante, los expertos especificaron que, como herramienta de orientación, permitiría que:

- Los profesionales supieran lo que se esperaba de ellos y pudieran realizar una autoevaluación de dónde se encontraban en relación con esta serie de capacidades. 
- Los estudiantes conocieran lo que el mercado demanda.

- Se redujera la brecha en las capacidades profesionales existente entre el graduado reciente y el profesional experimentado.

- Se entendiera que la organización es un actor social de gran responsabilidad que debe buscar proactivamente los temas y gestionarlos.

- Se contribuyera a la construcción curricular.

- Se lograra un rendimiento profesional más estandarizado en todo el mundo.

Como corolario de las fases del estudio, y considerando todas las contribuciones -particularmente las sugerencias realizadas en los grupos focales relativas a las superposiciones y categorías no exclusivas encontradas, al consenso obtenido en torno a la falta de algunas capacidades en la primera lista y a la redacción ambigua presentada en otras-, se estableció un listado final de capacidades del capítulo argentino de esta investigación global (ver tabla 13).

Tabla 13. Marco final de capacidades. Argentina

\begin{tabular}{|l|}
\hline $\begin{array}{l}\text { A. Entender el entorno social, económico, político y cultural de la organización/cliente y su negocio/actividad y } \\
\text { anticipar y predecir diferentes escenarios }\end{array}$ \\
\hline $\begin{array}{l}\text { B. Generar relaciones a largo plazo con los públicos de interés basadas en la confianza, la comunicación efec- } \\
\text { tiva y el consenso, para promover a la organización como un actor social responsable }\end{array}$ \\
\hline $\begin{array}{l}\text { C. Formular estrategias de comunicación que contribuyan al logro de los objetivos de la organización (objeti- } \\
\text { vos empresariales, sociales o de política pública) }\end{array}$ \\
\hline D. Actuar sobre la base de principios éticos, valores de inclusión social y diversidad \\
\hline E. Saber negociar, relacionarse con empatía y persuasión, incluso en momentos críticos \\
\hline F. Conocer el estado del arte de las tecnologías, canales y plataformas de comunicación y cómo utilizarlas \\
\hline G. Generar contenido a través de plataformas tradicionales y digitales e informes profesionales \\
\hline $\begin{array}{l}\text { H. Reconocer, gestionar y comunicar la identidad y los valores de la organización para promover su activo } \\
\text { reputacional }\end{array}$ \\
\hline $\begin{array}{l}\text { I. Desarrollar una formación profesional continua a lo largo de toda la carrera, que permita al profesional cono- } \\
\text { cer las tendencias y anticiparse a los cambios }\end{array}$ \\
\hline J. Establecer un sistema fiable de planificación y evaluación para medir el progreso y alcance de los objetivos \\
\hline K. Ser flexible y adaptable al cambio constante y a la presión \\
\hline
\end{tabular}

Fuente: elaboración propia.

\section{Discusión y conclusiones: análisis comparado}

Definir y concretar en formulaciones específicas y sintéticas cuál es el potencial de la profesión, y darle forma mediante el resultado de un marco, es siempre un reto difícil. La investigación llevada a cabo en el Global Capability Framework permite abrir una serie de discusiones acerca de la necesidad de pensar más allá del presente competencial para advertir cómo queremos dibujar al profesional del mañana.

Una profesión cambiante, que debe adaptarse para servir a las organizaciones en contextos inciertos, permite plantearse, como lo han hecho los expertos líderes seleccionados para la investigación, qué podría ser capaz de hacer un profesional para convertirse en un rol esencial en sus organizaciones y legítimo en la sociedad. 
Del análisis comparado de los marcos de capacidades resultantes en España y Argentina se coligen varias consideraciones que los autores presentan desde tres ángulos de análisis: 1) apreciaciones sobre la pertinencia de un marco de capacidades; 2) capacidades profesionales coincidentes que traspasan fronteras, y existencia de diferentes concepciones; y 3) qué futuro tiene pensar en el concepto de capacidad profesional, cuál puede ser su utilidad práctica.

Cabe decir, como apreciación general previa, que los hallazgos empíricos en Argentina y España muestran similitudes en muchos aspectos y algunas significativas diferencias, que se especifican en los siguientes tres puntos.

\section{1) Apreciaciones sobre la pertinencia de definir un marco de capacidades}

En ambos países, incluso siendo contextos diferentes y desarrollos históricos igualmente distintos, sí se aprecia una gran coincidencia sobre la necesidad de definir un marco de capacidades. Tal y como se observa en los apartados anteriores, tanto profesionales como académicos se han enfrentado al reto de definir un marco que representara la esencia y potencial desarrollo de la profesión; y esta cuestión ha sido acogida con mucho interés entre los expertos líderes de la muestra.

En ambos países se ha observado un interés unánime por adoptar esta perspectiva porque, a su juicio, facilita el diálogo sobre cuál es la función profesional; en definitiva, una perspectiva macro que enriquece el enfoque específico de niveles competenciales. Además, también se ha llegado a la misma apreciación en ambos países: un marco de capacidades es una fotografía futura que permite proyectar cuál es la misión profesional; $y$, por otro lado, otorga un mapa por donde transitar para diseñar la capacitación permanente de los profesionales en dos niveles: individual y gestión de equipos.

\section{2) Capacidades coincidentes que traspasan fronteras, y existencia de diferentes concepciones}

En ambos casos la capacidad coincidente es sobre el papel estratégico y de gobernanza organizacional que tiene la función comunicativa, aunque aún sea una asignatura pendiente, tal como está siendo evidenciado en estudios longitudinales como el de Moreno et al. (2017). Es decir, también ha emergido en esta investigación la preocupación por las capacidades que demostraran la dimensión estratégica, de asesoramiento y liderazgo interno de sus profesionales. Este hecho lo prueba que, en el caso de Argentina, seis de sus once capacidades del marco final están así orientadas (las identificadas con las letras A, B, C, G, H y J). Igualmente, en el caso de España, lo son cinco de sus nueve capacidades (como son la A, B, C, D e I).

Otra coincidencia en ambos países es considerar la comunicación como una función que contribuye a asegurar una adecuada lectura o análisis del contexto socio-político que rodea a las organizaciones. Este hecho se subraya en España con las capacidades C -ser consejero y asesor confiable- y D -contribuir a la gestión de relaciones con grupos de interés- y en Argentina con la capacidad A -entender el entorno para anticipar y predecir escenarios- y B -generar relaciones a largo plazo-. Éstas apuntan al corpus de conocimiento en las teorías de relaciones públicas, sobre la dimensión relacional de las organizaciones para que éstas consoliden su legitimidad social (cfr. la teoría dialógica y gestión relacional de las relaciones públicas). Tal y como afirman Gregory y Willis (2019: 113-124), la que denominan como inteligencia contextual de las organizaciones es una capacidad crítica de los comunicadores para contribuir a otras capacidades mencionadas en los marcos de ambos países: en España, las capacidades A -diseñar planes y estrategias alineadas-, B -apoyar en la gestión de intangibles- y E -ser conciencia crítica y ética de la organización-; o en Argentina las capacidades C -formular estrategias comunicativas para los objetivos organizacionales-y D -sobre los valores de inclusión social y diversidad-.

Como se aprecia en los hallazgos, las capacidades que han aportado los expertos en ambos países han coincidido en subrayar la dimensión de asesoramiento, estrategia y planificación, la gestión 
de relaciones con múltiples públicos y la opción de ser una conciencia crítica en la organización y asegurar comportamientos éticos y de inclusión social. En otro ángulo, también en España y Argentina los expertos han coincidido en señalar la importancia de los desafíos digitales y de múltiples plataformas y de la generación de contenidos.

Por otro lado, existen diferencias entre los marcos finales de capacidades en ambos países. En el caso español, se aprecia un interés preeminente por las cuestiones intangibles en la gestión organizativa; tanto es así que los expertos, tanto en el panel Delphi como en los grupos de discusión, han subrayado la necesidad de que en el futuro los profesionales fueran capaces de asesorar en estos aspectos mediante el desarrollo de métricas claras y más apego a la cultura del número. Por su parte, en el contexto argentino, los expertos se han diferenciado en su debate respecto de España acerca de las capacidades de formación profesional continua, que apenas se mencionaron, focalizando la discusión en aspectos organizativos. En Argentina, por su parte, se destaca la flexibilidad ante el cambio y la explicitación del ámbito diverso en lo sectorial en que las relaciones públicas y sus profesionales actúan: gubernamental, privado y social.

3) ¿Qué futuro y utilidad práctica tiene pensar en capacidades profesionales?

El debate sobre la utilidad de un pensamiento basado en capacidades profesionales ha sido acentuado por los expertos consultados en ambos países. En ambos casos, los investigadores tuvieron que explicitar y definir qué es el concepto de capacidad; diferenciarlo del planteamiento competencial, tanto en el panel Delphi como en los grupos focales, fue uno de los retos de la investigación. Los académicos y profesionales consultados han insistido en ambos países que es difícil explicitar las capacidades en planteamientos o frases sin pensar en las competencias que le subyacen. Sin embargo, en la propia discusión, como se ha visto, ha emergido un marco de capacidad porque los expertos de los paneles Delphi en ambos países han hecho un esfuerzo por sintetizar y abstraer cuáles son las capacidades esenciales que, a su juicio, constituyen una primera aproximación. Tal es así que sirvió como herramienta y planteamiento fundamental para que las posteriores discusiones en los grupos focales se facilitara, precisamente, la perspectiva de capacidades.

Al respecto, ésta es la primera conclusión destacada: los expertos consultados en los paneles Delphi y los grupos de discusión precisamente subrayaron que enmarcar la discusión en el concepto de capacidad les obligaba a dejar a un lado las competencias que hoy creen necesarias, para centrarse en discutir cuáles serán las capacidades que configuren al profesional del mañana.

Por otro lado, otra coincidencia en ambos países, más destacada y que centró gran parte de las discusiones en España, es que lograr la definición de un primer marco de capacidades profesionales supone una carta de presentación de la profesión como tal. Esto tiene varias consecuencias prácticas: en primer lugar, un marco de capacidades ayuda a que la profesión tenga una interlocución social más clara sobre su misión, de tal modo que podría contribuir a que sus profesionales supieran qué se espera de ellos, qué responsabilidades asumen y cómo debe capacitarse a los futuros profesionales. Lo último tiene indudables consecuencias en el diseño curricular de los estudios universitarios que, a diferencia de Argentina, en España se considera crucial dado que no está generalizada una oferta docente específica para la profesión. Es un tema éste que no ha sido objeto de análisis preferente en esta investigación, pero que podría considerarse una siguiente línea de investigación, entre otras, tal y como se destaca en el siguiente y último apartado.

\section{Limitaciones y futuras líneas de investigación}

Los hallazgos sobre capacidades en España y Argentina reflejan una visión sobre el potencial de sus profesionales centrada en aspectos estratégicos y sociales, y preocupados éstos por el valor que en el futuro la disciplina de la comunicación tiene que seguir ofreciendo para el buen hacer de sus 
organizaciones, en términos de diálogo social con los grupos de interés y para presentar una narrativa relevante en la esfera pública o social. Es así que los hallazgos de la metodología cualitativa implementada en ambos países permiten aseverar cuáles son las preocupaciones más comunes, y sus porqués, entre una muestra cualificada de expertos (profesionales y académicos). Sin embargo, una limitación de los hallazgos del estudio presentado es que no pueden extrapolarse estadísticamente, pues no se ha buscado tanto una representatividad estadística como construir un marco mediante una consulta a expertos, gracias a dos métodos cualitativos, pues ambos permiten canalizar la co-creación de contenidos entre los sujetos de la muestra.

Otra de las limitaciones de este trabajo, por su finalidad comparativa y lugar de origen y financiamiento del estudio, reside en que el marco teórico y la metodología se centran en perspectivas de escuelas anglosajonas en función de la intención explícita de construir un marco global de capacidades profesionales, tal y como se ha señalado en el segundo apartado sobre el origen del concepto en los años 80. En similar sentido, al permitir un margen de maniobra en los métodos de recopilación de datos para admitir variaciones culturales y contextuales, el estudio no pretende ser representativo ni efectuar comparaciones científicas estrictas, con lo cual los resultados obtenidos y las conclusiones extraídas no pueden extrapolarse directamente a la realidad profesional de otros países que no formaron parte del estudio. De todas formas, la expectativa es que este trabajo pueda ser de utilidad para otros que, a futuro, empleen una metodología similar en otros contextos nacionales.

En materia prospectiva y futuras líneas de investigación, en línea con el último aspecto mencionado, es deseable que la metodología y los resultados surgidos de este trabajo sean replicados por parte de académicos localizados en otros países iberoamericanos, para desarrollar capítulos nacionales, contribuyendo a extender la comparativa en lo regional y la condición inicial internacional del estudio original comenzado en nueve países. Esta investigación, a su vez, puede ofrecer resultados de interés para los profesionales de las relaciones públicas -de cara a su actualización permanente-, para los profesionales del área de recursos humanos -en pos de los procesos de reclutamiento y desarrollo profesional- y para los gestores académicos de carreras de relaciones públicas y comunicación -ante futuras actualizaciones curriculares en la lógica de las llamadas matrices de competencias-.

\section{Bibliografía}

Álvarez Nobell, A., Sadi, G. y Méndez, V. (2016). La institucionalización de la investigación en comunicación institucional y relaciones públicas en la Argentina. En Mateos, C. \& Herrero, J. (coord.) La pantalla insomne (2 $2^{\mathrm{a}}$ ed.). Cuadernos Artesanos de Comunicación, 103. https://doi. org/10.4185/cac103.

Barbour, R. (2008). Doing focus groups. London: Sage Publications.

Bartram, D. (2005). The Great Eight Competencies: A Criterion-Centric Approach to Validation. Journal of Applied Psychology, 90(6), 1185-1203. https://doi.org/10.1037/0021-9010.90.6.1185

Berkowitz, D. y Hristodoulakis. (1999). Practitioner Roles, Public Relations Education, and Professional Socialization: An Exploratory Study. Journal of Public Relations Research, 11(1), 91-103.

Broom, G. M. y Dozier, D. M. (1986). Advancement for public relations role models. Public Relations Review, 12(1), 37-56. https://doi.org/10.1016/S0363-8111(86)80039-X

Broom, G. M. y Smith, G. D. (1979). Testing the practitioner's impact on clients. Public Relations Review, 5(3), 47-59. 
Carbone, C. y Montaner, M. (2014). Argentina. En Watson, T. (Coord.). Latin American and Caribbean Perspectives on the Development of Public Relations: Other Voices (pp. 5-16). Palgrave Pilot. https://doi.org/10.1057/9781137404329

Commission on Public Relations Education (2017). Fast forward. Foundations + Future state. Educators - Practitioners. The Commission on PR Education 2017 Report on Undergraduate Education. Consultado en: http://www.commissionpred.org/

Delbecq, A. L., Van de Ven, A. H. y Gustafson, D. A. (1975). Group techniques for program planning. Scott Foresman.

Desanto, B. y Moss, D. (2005). Rediscovering what PR managers do: Rethinking the measurement of managerial behaviour in the public relations context. Journal of Communication Management, 9(2), 179-196. http://dx.doi.org/10.1108/13632540510621371

Dozier, D. M. (1992). The organizational roles of communications and public relations practitioners. En Grunig, J. E. (Ed.), Excellence in public relations and communication management (pp. 327-355). Mahwah NJ: Lawrence Erlbaum Associates.

Dozier, D. M. y Broom, G. M. (2006). The centrality of practitioner roles to public relations theory. En C. Botan y V. Hazleton (Eds.), Public Relations Theory II (pp. 137-170). Lawrence Erlbaum Associates.

Edwards, L. (2018). Understanding public relations. Theory, culture and society. London: Sage Publications.

Fawkes, J., Gregory, A., Falkheimer, J., Gutiérrez-García, E., Halff, G., Rensburg, R., ... Wolf, K. (2018). A Global Capability Framework for the Public Relations and Communication Management profession. Global Alliance for PR and Communication Management Research Report. Huddersfield, UK: University of Huddersfield.

Fawkes, J., Gregory, A. y Montoya-Martínez, E. (2017a). Rethinking competence and capability approaches, applied to public relations. In European Academy of Management (Ed.), Making knowledge work (pp. 1-36). University of Strathclyde.

Fawkes, J., Gregory, A., Montoya-Martínez, E. y Gutiérrez-García, E. (2017b). Intervening in public relations, competence and capability. En Interventions: Communication Research and Practice. Public Relations Theory (pp. 1-27). San Diego: 67th International Annual Conference, ICA (International Communication Association).

Ferrari M. y França. (2011). Relaciones públicas. Naturaleza, función y gestión en las organizaciones contemporáneas. Buenos Aires: La Crujía.

Global Alliance for Public Relations and Communication Management. (2016). From the GBOK to a Global Capability Framework. Lugano. Consultado en: https://static1.squarespace. com/static/561d0274e4b0601b7c814ca9/t/574ae46707eaa010521f0d31/1464525936413/08_ GBOK+Progress+report+\%26+global+capabilities+framework+May+2016.pdf

Global Alliance for Public Relations and Communication Management (2015). Global Body of Knowledge (GBOK) Project. "The standard" to practice public relations and communication management. Lugano. Consultado en: https://static1.squarespace.com/ static/561d0274e4b0601b7c814ca9/t/56c1 faea59827e4bccf4daf2/1455553260847/GBOK2015.pdf

Gregory, A. (2008). Competencies of senior communication practitioners in the UK: An initial study. Public Relations Review, 34(3), 215-223. https://doi.org/10.1016/j.pubrev.2008.04.005 
Gregory, A. y Fawkes, J. (2019). A global capability framework: Reframing public relations for a changing world. Public Relations Review, 45(3), 101781. https://doi.org/10.1016/j.pubrev.2019.05.002

Gregory, A. y Willis, P. (2019). Liderazgo estratégico y gestión de la comunicación. Baranáin: EUNSA y Biblioteca Corporate Excellence.

Gutiérrez-García, E. y Recalde, M. (2018). Dibujando al profesional del mañana. Estudio Delphi sobre capacidades de la comunicación estratégica en España. Analisi-Quaderns de Comunicacio I Cultura, 59, 1-18. https://doi.org/10.5565/rev/\%0Aanalisi.3153

Gutiérrez-García, E. y Rodríguez Salcedo, N. (2009). 50 años de Relaciones Públicas en España. De la propaganda y la publicidad a la gestión de la reputación. Doxa Comunicación, 9, 13-33.

Krueger, R. A. (1998). Analyzing \& reporting focus groups results. Sage Publications.

Lester, S. (2009). Routes to qualified status: practices and trends among UK professionals bodies. Studies in Higher Education, 43(2), 223-236.

Lester, S. (2014). Professional standards, competence and capability. Higher Education, Skills and Work-Based Learning, 4(1), 31-43. https://doi.org/10.1108/HESWBL-04-2013-0005

Linstone, H. A. y Turoff, M. (1975). The Delphi method: techniques and applications. Reading, Mass., Mass.: Addison-Wesley.

Manley, D. y Valin, J. (2017). Laying the foundation for a global body of knowledge in public relations and communications management. Public Relations Review, 43(1), 56-70. https://doi. org/10.1016/J.PUBREV.2016.10.018

Montero, M., Rodríguez Salcedo, N. y Verdera, F. (2010). Historia de la publicidad y de las relaciones públicas en España. De la nada al consumo: de los orígenes hasta 1960. Madrid: Comunicación Social Ediciones y Publicaciones.

Moreno, A. (2004). Spain. En B. van Ruler y D. Verčič (Eds.). Public relations and communication management in Europe : a nation-by-nation introduction to public relations theory and practice (pp. 393-412). Mouton de Grouter.

Moreno, A., Molleda, J. C., Athaydes, A., Suárez, A. M., Herrera, M. y Álvarez, A (2017). Latin American Communication Monitor 2016-2017. Tendencias en comunicación estratégica: big data, automatización, engagement, influencers, coaching y competencias. Resultados de una encuesta en 17 paises. Madrid: EUPRERA/DIRCOM.

Moreno, A., Tench, R. y Okay, A. (2017). Re-fuelling the talent tank. A qualitative study of key deficiencies, future needs, and life-long learning needs of communication management professionals in Europe. Communication \& Society, 30(3), 109-127.

Moss, D., Newman, A. y DeSanto, B. (2005). What do Communication Managers Do? Defining and Refining the Core Elements of Management in a Public Relations/Corporate Communication Context. Journalism \& Mass Communication Quarterly, 82(4), 873-890. https://doi. org/10.1177/107769900508200408.

Nothhaft, H. (2016). A Framework for Strategic Communication Research: A Call for Synthesis and Consilience. International Journal of Strategic Communication, 10(2), 69-86.

Nussbaum, M. C. (2004). Beyond the social contract: capabilities and global justice. Oxford Development Studies, 32(1), 3-18. https://doi.org/10.1080/1360081042000184093 
O’Reilly, D., Cunningham, L. y Lester, S. (Eds.). (1999). Developing the capable practitioner: Professional capability through higher education. Nueva York: Kogan Page.

Robeyns, I. (2005). The Capability Approach: a theoretical survey. Journal of Human Development, 6(1), 93-117. https://doi.org/10.1080/146498805200034266

Rodríguez-Salcedo, N. (2015). Contributions to the History of Public Relations in the Midst of a Dictatorship: First Steps in the Professionalization of Public Relations in Spain (19601975). Journal of Public Relations Research, 27(3), 212-228. https://doi.org/10.1080/106272 6X.2015.1024248

Rodríguez Salcedo, N. (2008). Public relations before "public relations" in Spain: an early history (1881-1960). Journal of Communication Management, 12(4), 279-293. https://doi. org/10.1108/13632540810919756

Rodríguez-Salcedo, N. y Watson, T. (2017). The development of public relations in dictatorships - Southern and Eastern European perspectives from 1945 to 1990. Public Relations Review, 43(2), 375-381. https://doi.org/10.1016/J.PUBREV.2017.01.001

Ruler, B. van, Vercic, D., Bütschi, G., \& Flodin, B. (2009). A First Look for Parameters of Public Relations in Europe. Journal of Public Relations Research, 16(1), 1-34.

Saavedra Llamas, M. y Perlado Lamo de Espinosa, M. (2017). Los estudios universitarios especializados en comunicación en España. Barcelona: UOC.

Sadi, G. (2014). En torno al objeto de estudio y los roles sociales de las relaciones públicas.

Organicom, Revista Brasileira de Comunicação Organizacional e Relações Públicas, 11(21), 31-44.

Sandhu, S. (2017). Heading for Mars While We Haven't Been on the Moon: A Reply to Nothhaft. International Journal of Strategic Communication, 11(3), 184-188. https://doi.org/10.1080/15531 18X.2017.1319837

Sen, A. (1993). Capability and Well-Being. En M. Nussbaum \& A. Sen (Eds.), The quality of life (pp. 30-53). Oxford: Oxford University Press.

Stephenson, J. (1998). The Concept of Capability and its Importance in Higher Education. En J. Stephenson y M. Yorke (Eds.), Capability \& Quality in Higher Education (pp. 1-13). Kogan Page.

Stewart, D. W. y Shamdasani, P. N. (1990). Focus groups. Theory and practice. Thousand Oaks: Sage Publications.

Tench, R., Zerfaß, A., Verhoeven, P., Verčič, D., Moreno, Á. y Okay, A. (2013). Competencies and role requirements of communication professionals in Europe. Insights from quantitative and qualitative studies. ECOPSI research report. Leeds.

Thurlow, A., Sévigny, A. y Dottori, M. (2018). Global Capabilities in Public Relations. Public Relations Journal, 11(3), 1-25.

Wakefield, R. y Watson, T. (2014). A reappraisal of Delphi 2.0 for public relations research. Public Relations Review, 40(3), 577-584. https://doi.org/10.1016/J.PUBREV.2013.12.004

Watson, T. (2008). Public relations research priorities: a Delphi study. Journal of Communication Management, 12(2), 104-123. https://doi.org/10.1108/13632540810881938 